\title{
Geometry of the bandaging procedure and its application while wrapping bandages for treatment of leg ulcers
}

\author{
Monica Puri Sikka*, Subrato Ghosh, Arunangshu Mukhopadhyay \\ Department of Textile Technology, National Institute of Technology, Jalandhar, India \\ Email: ${ }^{*}$ sikkamonica@yahoo.co.in, ghoshs@nitj.ac.in, arunangshu@nitj.ac.in
}

Received 7 October 2013; revised 15 November 2013; accepted 28 November 2013

Copyright (C) 2013 Monica Puri Sikka et al. This is an open access article distributed under the Creative Commons Attribution License, which permits unrestricted use, distribution, and reproduction in any medium, provided the original work is properly cited. In accordance of the Creative Commons Attribution License all Copyrights (C) 2013 are reserved for SCIRP and the owner of the intellectual property Monica Puri Sikka et al. All Copyright (C) 2013 are guarded by law and by SCIRP as a guardian.

\begin{abstract}
Appropriate compression bandaging is important for compression therapeutic medical diseases. The high compression approach employed for treating venous leg ulcers should be used correctly so that sufficient (but not excessive) pressure is applied. Bandages used to treat venous disease by compression should achieve and sustain effective levels and gradients of pressure and minimize the risk of pressure trauma. To maintain graduated compression on the limb, the bandage needs to be applied at the same tension for each layer from the ankle to the knee. In this paper, the geometry for various bandaging procedures is used to wrap each layer of bandage by marking the relaxed length of the bandage. The relaxed length is calculated depending on the stretch\%, the average circumference of the limb to which it is to be applied and the bandaging technique to be used. This paper aims at developing a scientific approach while applying the bandage to reduce the inter operator variability in applying the same tension on each successive layer of bandage.
\end{abstract}

Keywords: Bandaging; Compression; Inter Operator Variability; Graduated; Relaxed Length; Stretch

\section{INTRODUCTION}

Compression therapy is used for the treatment of the venous leg ulceration and for other chronic venous insufficiency $[1,2]$. Compression is provided by wrapping the bandage around the limb by the application of external force. Because of compression, the pressure is generated at the interface between bandage and skin and this pressure is called interface pressure or sub-bandage pressure.

\footnotetext{
${ }^{*}$ Corresponding author.
}

The efficiency of the treatment depends to a great degree on the level of interface pressure applied and sustenance of this pressure during the course of the treatment. Provided that the right level of interface pressure does not affect arterial flow and the right level of application technique and materials used, the effects of compression can be dramatic, which could reduce oedema and pain and also could promote healing of ulcers caused by venous insufficiency $[3,4]$. So compression bandaging requires skill, appropriate training and initial supervision of practice [5].

The degree of compression produced by any bandage system over a period of time is determined by complex interactions between four principle factors - the physical structure and elastomeric properties of the bandage, the size and shape of the limb to which it is applied, the skill and technique of the bandager and the nature of any physical activity undertaken by the patient [6]. It is essential that practitioners understand how application techniques can affect the performance of the bandage systems [7]. Inappropriate selection or application of a bandage could lead to lack of efficacy and to adverse effects including amputation [8]. Lee et al. [9] observed the importance of different application techniques on the interface pressure variations for different bandages. Wrapping of bandage over wounded limb by different practitioners could also influence interface pressure variation. Dale et al. [10] observed different pressure gradients obtained by the same bandaging system when applied by different experienced technicians under the same application technique.

Many attempts have been made to reduce the effects of operator variability by marking bandages with geometrical shapes that change from rectangles to squares or from ovals to circles when a particular level of extension has been applied [11]. Not all bandages have these geometrical shapes and some manufactures ask users to ap- 
ply medical compression bandages with a percentage of extension [11]. Without any visual markers, the real stretch obtained on application of bandage by qualified personnel can vary from $35 \%$ to $70 \%$, leading to a risk of under or over-application of pressure [12].

The geometrical shapes used for applying the desired stretch $\%$ on the bandages have a few drawbacks. The change in shape is visual and depends on individual perception, so there is no guarantee that the recommended stretch $\%$ is maintained. Also there is always a range given by the manufacturer for the pressure values and the limb circumference for a particular stretch\% value. Furthermore, these values are given for only one type of bandaging procedure that generally spirals $50 \%$ overlap. Finally it has been seen while applying such a type of bandage that the rectangles change into squares at different stretch values than what is recommended by the manufacturer.

An experimental set up was done to see the change of shape on the bandage at different stretch levels as recommended by the manufacturer for a bandage. The sample is clamped at two edges and a weight equivalent to the stretch\% value is hung at the bottom and then the change in shape is observed. The best fit linear equation for the load elongation curve of the bandage (generated using Excel) was used to estimate the load for the given stretch $\%$ value (Figure 1). The weights hung according to the recommended stretch $\%$ for green rectangles and brown rectangles in the bandage reveal that a small change in weight has no visible effect on the shape but the corresponding change in stretch $\%$ and thus the sub bandage pressure could be of concern.

To cater to these problems and to provide a scientific approach to wrap a bandage at a particular level of stretch for each layer, a geometrical model is proposed. The model is developed for two commonly used techniques of bandaging i.e. spiral bandaging and ascending spica or figure of eight bandaging [13].

\section{GEOMETRY OF THE BANDAGING PROCEDURE}

The pressure on the limb is developed by the tension in the bandage fabric and this tension is the result of the

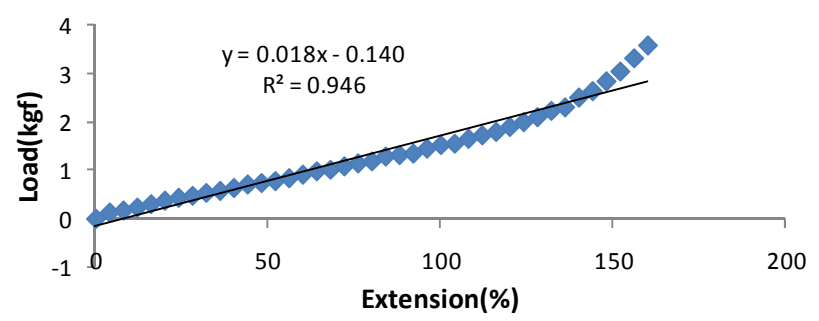

Figure 1. Best fit linear equation for the load extension curve of the bandage. stretch/force applied in the fabric. To provide a uniform stretch in each layer, the limb circumference and fabric thickness are to be linked to the stretch in the fabric for generating pressure in the limb. When the bandage fabric is applied layer by layer on the cylindrical body; the circumference of the cylindrical body increases due to the fabric thickness so in order to wrap all the layers of bandages with uniform stretch (\%), a relationship between these three parameters is necessary. The relationship has been formulated [14] and given below. The actual length of bandage sample in relaxed state $(l)$ is to be stretched to an extra amount of length to be wrapped on cylindrical body having circumferential length $(c)$, to get the predefined stretch level $(S \%)$ for $100 \%$ overlap, which is given by:

$$
l=\frac{c}{1+\frac{s}{100}}=\frac{2 \pi[r+(n-1) \times t]}{1+\frac{s}{100}}
$$

where $n=$ number of layers of the bandage, $t=$ thickness of the bandage, $r=$ radius of curvature of the cylinder surface.

Taking this further and extending to spiral bandaging and figure of eight bandaging a relationship has been developed between fabric stretch, circumference of bandaging surface and bandage fabric thickness considering the simplified geometry of the two types of bandaging techniques. The assumptions taken are:

1) The surface of the cylinder is uniform throughout.

2) The stretch percentage all along the fabric length is the same.

3) Thickness of the bandage is measured in standard thickness testing condition.

The spiral bandaging (Figure 2) on a uniform circular cylinder can be represented geometrically as in Figure 3 and further this cylinder is flat opened (Figure 4).

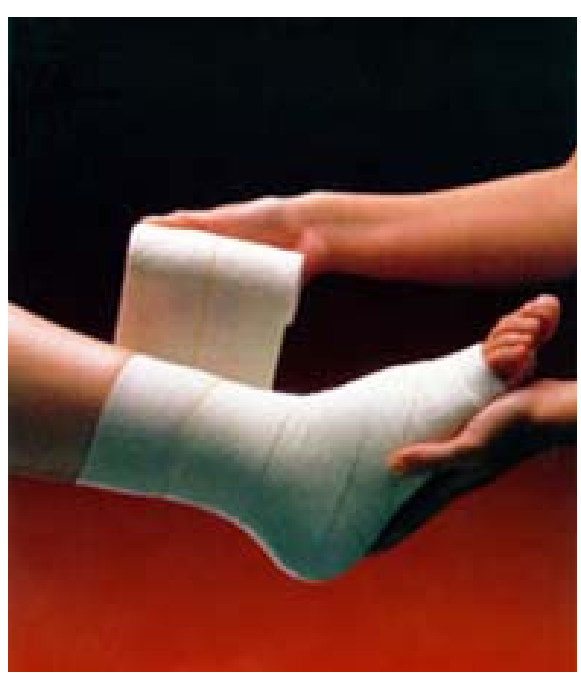

Figure 2. Spiral bandaging [13]. 


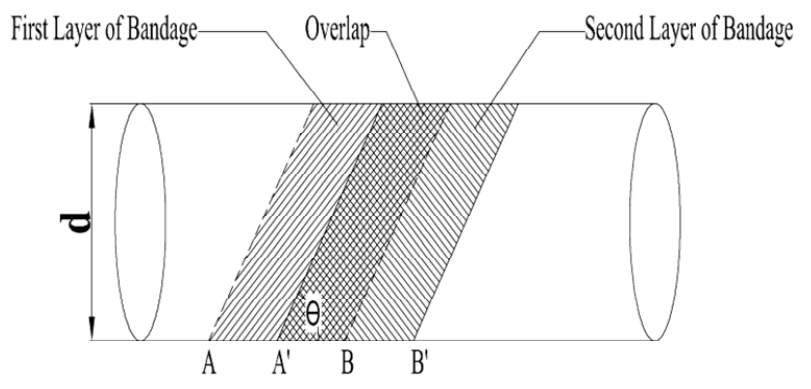

Figure 3. Geometry of spiral bandaging.

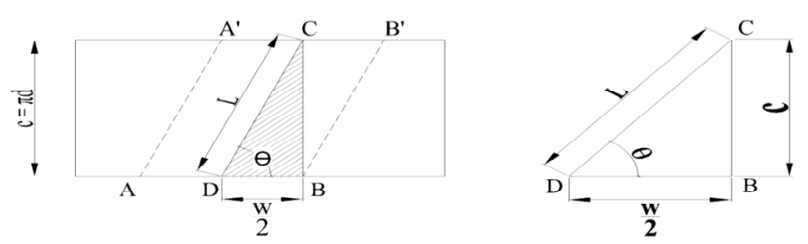

Figure 4. Flattened cylindrical view for spiral bandaging.

where $c=$ circumference of the cylindrical body in "cm", $d=$ diameter of the cylinder, $t=$ thickness of the bandage in "cm", $r=$ radius of curvature of the cylinder surface, $w$ $=$ width of bandage in "cm".

Now considering the triangle CBD (Figure 4), the circumferential length $(L)$ can be expressed as:

$$
L=c \operatorname{cosec} \theta=c \sqrt{1+\cot ^{2} \theta}
$$

where $\theta=$ angle of bandaging, $c=$ circumference $=\pi d$.

When the bandage of width " $w$ " $\mathrm{cm}$ is wrapped spirally with $50 \%$ overlap, from the triangle CBD (Figure 4).

$$
\begin{gathered}
\tan \theta=\frac{c}{w / 2}=2 c / w \\
\cot \theta=w / 2 c
\end{gathered}
$$

Putting the value of $\cot \theta$ from Eq.3 in Eq.2

$$
\begin{aligned}
& L=c \sqrt{1+(w / 2 c)^{2}} \\
& L=\frac{1}{2} \sqrt{4 c^{2}+w^{2}}
\end{aligned}
$$

Similarly for spiral $66 \%$ overlap, $\tan \theta=3 c / w$, so

$$
L=\frac{1}{3} \sqrt{9 c^{2}+w^{2}}
$$

And for spiral $100 \%$ overlap since $\theta=90^{\circ}, \cot \theta=0$, hence $L=c$.

For spiral bandaging both $50 \%$ and $66 \%$ overlap " $c$ " is replaced by the new circumferential length " $L$ " in Eq.1. Substituting the value of L from Eq.4 in Eq.1, actual length of bandage fabric $(l)$ to be taken in relaxed state to apply it spirally at $50 \%$ overlap at the predefined stretch level can be calculated in terms of $r, n, t, w, S$ which are the known parameters to generate a particular stretch value Eq.6.

$$
\begin{aligned}
l & =\frac{L}{1+\frac{s}{100}}=\frac{\frac{1}{2} \sqrt{4 c^{2}+w^{2}}}{1+\frac{s}{100}} \\
& =\frac{1 / 2 \sqrt{4[2 \pi\{r+(n-1) t\}]^{2}+w^{2}}}{1+\frac{S}{100}}
\end{aligned}
$$

Similarly the length of bandage fabric $(l)$ in relaxed state to be taken to apply it spirally at $66 \%$ overlap and any predefined stretch level can be calculated in terms of $r, n, t, w, S$ from Eq.7

$$
l=\frac{L}{1+\frac{S}{100}}=\frac{\frac{1}{3} \sqrt{9 c^{2}+w^{2}}}{1+\frac{S}{100}}
$$

So

$$
l=\frac{1 / 3 \sqrt{9[2 \pi\{r+(n-1) t\}]^{2}+w^{2}}}{1+\frac{S}{100}}
$$

To calculate the length of bandage fabric $(l)$ in relaxed state for figure of eight bandaging technique, let the bandage be applied in figure of eight at 50\% overlap (Figure 5). Figure 6 is the geometrical representation of the figure of eight bandaging technique on a uniform circular cylindrical limb. When the cylinder is flat opened, it can be represented as shown in the Figure 7.

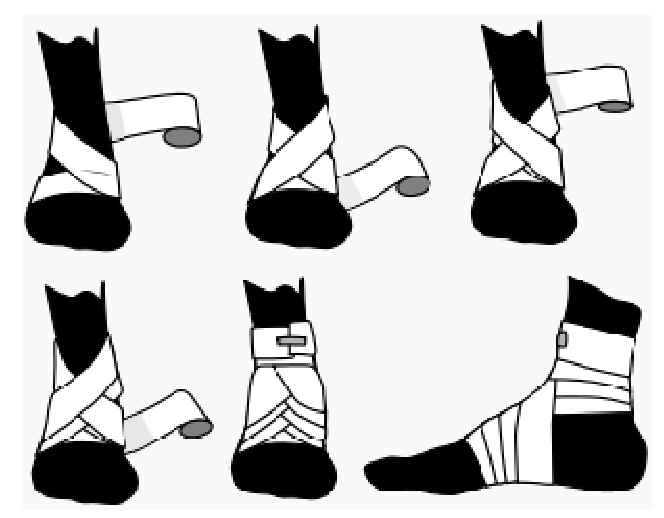

Figure 5. Figure of eight bandaging [13].

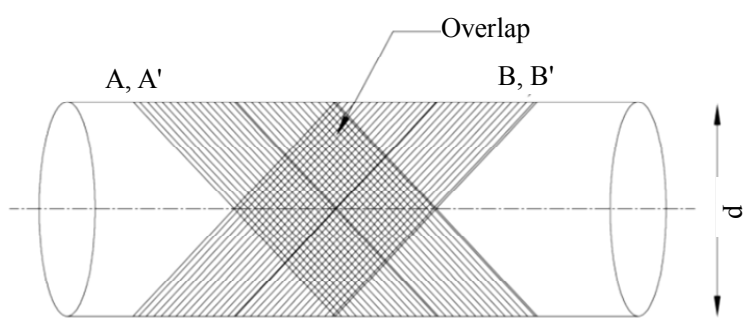

Figure 6. Geometry for figure of eight bandaging. 
From the triangle QA'R in Figure 7

$$
(L / 4)^{2}=(p / 2)^{2}+(c / 2)^{2}
$$

where $L=$ new circumferential length, $w=$ width of the bandage, $p=$ spacing between the two extreme turns of the bandage and its value is always between $w$ and $2 w$

$$
\begin{aligned}
& \frac{L^{2}}{16}=\frac{p^{2}}{4}+\frac{c^{2}}{4} \\
& L^{2}=4 p^{2}+4 c^{2} \\
& L=2 \sqrt{p^{2}+c^{2}}
\end{aligned}
$$

For figure of eight bandaging 50\% overlap " $c$ " in Eq.1 is replaced by the new circumferential length " $L$ ". Now putting the value of " $L$ " from Eq.8 in Eq.1, the actual length of bandage fabric $(l)$ in relaxed state to be applied in figure of eight at $50 \%$ overlap is given by:

$$
\begin{gathered}
l=\frac{L}{1+\frac{S}{100}} \\
l=\frac{2 \sqrt{p^{2}+[2 \pi\{r+(n-1) t\}]^{2}}}{1+\frac{S}{100}}
\end{gathered}
$$

The value of $p$ for figure of eight bandaging with $50 \%$ overlap as experimentally measured after application is one and a half times (1.5) the width of the bandage. The second overlap in figure of eight bandage is exactly halfway the width of the first bandage overlap in addition to one full width of the bandage.

\section{APPLICATION OF THE GEOMETRICAL MODEL IN BANDAGING PRACTICE}

The geometrical model calculates the relaxed length of

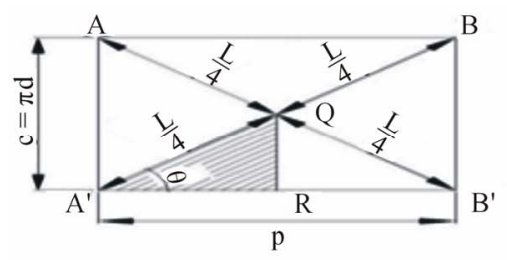

bandage to be wrapped on a given limb to maintain a particular stretch for a given circumference and number of layers depending on different bandaging techniques. To illustrate this, the value of relaxed length " $l$ " is calculated layer wise using different values of stretch\% and the number of layers for different types of bandaging techniques taking $38.5 \mathrm{~cm}$ circumference of the leg and $0.834 \mathrm{~mm}$ bandage thickness according to the Eq.6, 7 and 9.

It is observed from the Table $\mathbf{1}$ that the relaxed length is much less than the actual circumferential length and it decreases further with increase in stretch value. Also as the number of layer increases, the bandaging circumference also increases and corresponding to this circumference, the relaxed length of bandage sample " $l$ " also increases at various stretch levels to get uniform stretch for each layer. This calculated relaxed length can be now marked on the bandage and wrapped layer wise to get the desired stretch $\%$ at each layer.

\section{CONCLUSIONS}

Practitioners require guidelines to follow that are evidence-based, relate to local needs and are easy to follow. The current compression therapy techniques do not provide users with sufficient and accurate feedback concerning the interface pressures applied by them. Moreover the geometrical shapes provided on the bandages by the manufacturers give a range of pressure values for a stretch $\%$ for one type of bandaging procedure (generally spiral $50 \%$ overlap). Also it is observed that these shape changes are dependant on individual visual perception.

To deal with these intricacies, this model has been developed based on the geometry of bandaging procedure

\begin{tabular}{|c|c|c|c|c|c|c|c|}
\hline \multirow{2}{*}{ Stretch $(\%)$} & \multicolumn{2}{|c|}{$\begin{array}{l}\text { Relaxed length of bandage " } l " \\
\text { for spiral } 50 \% \text { overlap }(\mathrm{cm})\end{array}$} & \multicolumn{2}{|c|}{$\begin{array}{l}\text { Relaxed length of bandage "l" } \\
\text { for spiral } 66 \% \text { overlap }(\mathrm{cm})\end{array}$} & \multicolumn{3}{|c|}{$\begin{array}{l}\text { Relaxed length of bandage "l" for } \\
\text { figure of eight } 50 \% \text { overlap }(\mathrm{cm})\end{array}$} \\
\hline & First layer & Second layer & First layer & Second layer & Third layer & $\begin{array}{l}\text { First and second } \\
\text { layer }\end{array}$ & $\begin{array}{c}\text { Third and fourth } \\
\text { layer }\end{array}$ \\
\hline $30 \%$ & 29.86 & 30.26 & 29.72 & 30.11 & 30.52 & 31.78 & 32.15 \\
\hline $40 \%$ & 27.73 & 28.10 & 27.60 & 27.95 & 28.34 & 29.51 & 29.86 \\
\hline $50 \%$ & 25.88 & 26.22 & 25.76 & 26.09 & 26.45 & 27.54 & 27.87 \\
\hline
\end{tabular}
which will not only effectively reduce the problem of inter operator variability during wrapping but also will

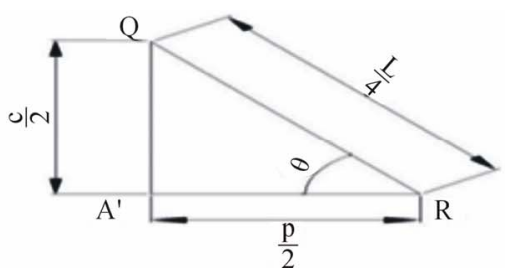

Figure 7. Flattened cylindrical view for figure of eight bandaging.

Table 1. Relaxed length of bandage layers for various bandaging techniques. 
cater to the need for generating graduated and consistent level of compression. The model has been developed with an intention to follow an appropriate bandaging process according to the desired stretch\% and the bandaging technique to be used.

\section{REFERENCES}

[1] Farah, R.S. and Davis, M.D.P. (2010) Venous leg ulcerations: A treatment updates. Current Treatment Options in Cardiovascular Medicine, 12, 101-116. http://dx.doi.org/10.1007/s11936-010-0066-9

[2] Vicaretti, M. (2010) Compression therapy for venous disease. Australian Prescriber, 33, 186-190.

[3] Clark, M. (2003) Compression bandages: Principles and definitions. Position Document, European Wound Management Association (EWMA), Medical Education Partnership Ltd., London, 2-4.

[4] Mosti, G., Mattaliano, V., Polignano, R. and Masina, M. (2009) Compression therapy in the treatment of leg ulcers, Acta Vulnologica, 7, 1-43.

[5] Nelson, E., et al. (1996) The management of leg ulcers. Journal of Wound Care, 5, 73-76.

[6] EWMA Position Document (2003) Understanding compression therapy. MEP, London. http://www.proguide.net

[7] Logan, R.A., Thomas, S. and Harding, E.F. (1992) A comparison of sub bandage pressures produced by ex- perienced and inexperienced bandagers. Journal of Wound Care, 1, 23-26.

[8] Thomas S, Nelson AE. (1998) Graduated external compression in the treatment of venous disease. Journal of Wound Care, 78, 1-4.

[9] Lee, A.J., Dale, J.J., Ruckley, C.V., Gibson, B., Prescott, R.J. and Brown, D. (2006) Compression therapy: Effects of posture and application techniques on initial pressures delivered by bandages of different physical properties. European Journal of Vascular and Endovascular Surgery, 31, 542-552. http://dx.doi.org/10.1016/j.ejvs.2005.10.023

[10] Dale, J.J., Ruckley, C.V., Gibson, B., Brown, D., Lee, A.J. and Prescott, R.J. (2004) Multi-layer compression: Comparison of four different four-layer bandage systems applied to the leg. European Journal of Vascular and Endovascular Surgery, 27, 94-99. http://dx.doi.org/10.1016/j.ejvs.2003.10.014

[11] Thomas, S. and Fram, P. (2003) An evaluation of a new type of compression bandaging system. www.worldwidewounds.com

[12] (2010) www.urgomedical.com

[13] Compression Therapy Study Group (2009) Compression, consensus document based on scientific evidence and clinical experience. Edizioni Minerva Medica Torino, Compression impa.indd 3.

[14] Venkata, P. (2011) Experimental and theoretical studies on compressive behaviour of crepe bandage fabrics. M.Tech thesis, N.I.T-Jalandhar, 1-60. 